\title{
Littérature et dissolution des genres artistiques
}

\author{
Wilfried Laforge \\ Université Lille III
}

Pour cette minuscule promesse de bonheur qui ne se perd pas en consolations, il a certes fallu payer un prix élevé: aller jusqu'au bout de l'articulation jusqu'à perdre tout contact avec le monde. Il faut dénoncer tout engagement en faveur du monde pour satisfaire à l'idée de l'œuvre engagée.

Adorno, Notes sur la littérature

Dans un essai de ses Notes sur la littérature, intitulé «la situation du narrateur dans le roman contemporain », Adorno nous dit du roman qu'il se caractérise désormais au moyen d'un 
paradoxe. En effet, il est devenu impossible de narrer : "Alors que la forme du roman exige la narration [...] raconter quelque chose, c'est avoir quelque chose de particulier à dire, et c'est justement ce qu'interdit le monde quadrillé, la standardisation et la répétition éternelle. » (1984, p. 37-38) Quelques années plus tard, dans L'Art et les arts, Adorno revient sur ce retrait du contenu narratif. Il observe que celui-ci peut être pallié, notamment, par des emprunts aux structures de la musique : tel est le cas, nous dit notre philosophe, chez Helms. Ce constat adornien constituera notre point de départ pour une première partie : nous le verrons, l'emprunt à visée palliative n'est pas le seul fait du roman ou de la littérature; nous pensons qu'il s'inscrit au contraire dans le cadre d'un bouleversement plus général au sein de la pratique artistique, qui n'est autre qu'une dissolution des genres artistiques au sein de laquelle la tentation d'emprunts aux structures des autres arts est omniprésente. Cette dissolution aboutira à l'effondrement complet des différents arts et à la possibilité de faire de « l'art en général » - en d'autres termes, sans être le praticien d'un genre en particulier - pour le dire avec les mots du critique d'art américain Clement Greenberg.

L'effondrement des limites entre les genres entraîne celui des limites qui séparent l'art de la vie. Par voie de conséquence, poser l'autonomie de l'art devient alors problématique : un tel énoncé suppose en effet l'indépendance de l'art vis-à-vis du réel, de tout ce qui lui est extrinsèque et n'obéit pas à ses règles propres, autrement dit, la conception d'un domaine de l'art cloisonné et distinct de toute autre activité humaine. Nous proposerons donc une brève analyse de la conception adornienne de l'autonomie de l'art, conception particulièrement riche et féconde pour penser le rapport de l'art au réel, dans la 
mesure où le souci de sauvegarder l'autonomie de l'art - la distance qui la sépare du réel et de la vie - , tout en conservant son aspect social, y est constant: il s'agit de sauver, complémentairement, et cette autonomie et cet ancrage dans la culture et la réalité empirique. Autrement dit, l'autonomie de l'art signifie, pour Adorno, son émancipation à l'égard de la théologie, de la métaphysique et de toute fonction cultuelle. Pour lui, s'il faut reconnaître à l'idéalisme le mérite d'avoir favorisé cette libération, il a en revanche renforcé le « caractère emphatique » de l'art. Celui-ci est en étroite liaison avec la présence ambiguë de l'art au monde, son affirmation et sa positivité. Ce caractère ambigu de l'art (Doppelchrakter der Kunst) est aussi dû au fait qu'il soit à la fois autonome et fait social, participant à la réalité empirique tout en s'en détachant : nous le verrons, chez Adorno, «l'œuvre d'art est rebelle par sa structure même » et " toute réconciliation avec le monde qu'elle dépeint est inimaginable. » (Marcuse, 1973, p. 119)

À l'aube des années 1960, on a le sentiment généralisé qu'après s'être trouvées mises à mal, les limites qui séparent les différents arts ont purement et simplement éclaté et qu'il est désormais possible d'être artiste sans être nécessairement le praticien d'un art en particulier: on entre dès lors dans un système totalement nouveau. Les années qui ont suivi ce qu'Adorno a proposé de nommer l'«effrangement » (Verfransung) des genres, puis leur abolition complète, ont confirmé les tendances mises en place dans les années 1960 et la légitimation des pratiques nouvelles alors lancées : les débats sur le concept d'art semblent avoir perdu de leur actualité. Lorsqu'on s'est mis à évoquer l'interdisciplinarité en art — avec des expressions telles que « mixed media », «multimedia art», 
etc. -, il s'agissait surtout de faire une place à des pratiques qui n'entraient plus dans les catégories traditionnelles.

Doit-on, aujourd'hui, s'inquiéter que les arts ne travaillent plus dans « le respect du médium » (Greenberg) ou encore dans « la contrainte du matériau » (Adorno)? Doit-on penser que la fin d'une différence des arts menace la capacité « sismographique » des œuvres d'art, pour le dire avec Adorno? Pour répondre à ces questions, il faut comprendre ce qui est en jeu dans cette dissolution des genres artistiques.

\section{Dissolution et « effrangement 》}

Revenons à notre constat de départ et tentons de le préciser. Bien qu'aux lendemains de la Seconde Guerre mondiale s'épanouisse, de ce côté de l'Atlantique, une certaine forme de modernité qui semble spécifiquement américaine, on l'a vu, à la fin des années 1960, critiques, historiens de l'art et philosophes s'accordent à dire qu'en Europe comme aux États-Unis, les limites entre les différents genres ont éclaté. Vers 1966, le débat sur cette dissolution des genres artistiques gagne les milieux universitaires, où l'on commence à s'interroger sur le concept d'art en général et sur le rapport des arts à l'art. Le sentiment généralisé est qu'il est désormais possible et, qui plus est, institutionnellement légitime, d'être artiste sans pratiquer nécessairement un art en particulier. Le travail sur l'institution, sa critique au sein même de la pratique artistique ou encore l'émergence de l'art conceptuel semblent alors constituer les symptômes puissants du passage à un système différent. Une tendance lourde se dessine; les artistes cessent de travailler selon des normes, des conventions, sous la "contrainte d'un 
matériau » spécifique, en suivant une tradition. Ou, mieux dit : les contraintes sont désormais personnelles et ceux qui veulent reconstituer une tradition ont recours à la filiation. Cet effondrement des limites entre les différents genres entraîne celui des limites qui séparent l'art de la vie. Bien que le constat semble unanime, les réactions sont, quant à elles, très diverses. D'aucuns adoptent une position eschatologique et pensent assister à la fin de l'art et au début d'un art " post-historique », comme Arthur Danto. D'autres, qui ne joignent pas leur voix aux lamentations sur la fin de l'art, alors maintes fois répétées, y voient un changement de définition de l'art et s'inquiètent que celui-ci perde sa capacité à "écrire l'histoire» et cesse de «crépiter» (Adorno). Ou encore ils s'alarment de voir les artistes de la nouvelle génération - épigones plus ou moins légitimes de Marcel Duchamp - abandonner progressivement leur genre au profit d'un art dé- et re-contextualisé, "dédéfini » (voir Rosenberg, 1991). Adorno est de ceux qui échappent aux débats pessimistes en montrant que c'est un concept spécifique d'art qui disparaît, et non l'art lui-même : la dissolution des genres n'est qu'un faux déclin de l'art. Pour Harold Rosenberg, ce déclin, l'abandon de la tradition - en tant que libération d'un passé qui poussait l'art dans une direction unique - plonge l'art dans un malaise permanent, indissolublement lié à l'angoisse du possible dont souffre tout homme libre. Mais ce qui n'est qu'un constat pour certains prend la forme d'une réaction enthousiaste chez d'autres. Sous la plume d'Allan Kaprow par exemple, lequel écrivait déjà en 1958 : « les jeunes artistes aujourd'hui n'ont plus besoin de dire je suis peintre, ou poète, ou danseur, ils sont simplement artistes ». À la même époque, certains théoriciens vont même jusqu'à prescrire le passage des arts — au pluriel — 
à l'art, à l'art « en général » (Greenberg). C'est le cas de Joseph Kosuth notamment, avec Art after philosophy, en 1969.

À la suite de ce constat, tentons d'analyser les spécificités de cette dissolution des genres, puis de l'abolition des limites entre les arts sur un plan historique et esthétique, en montrant notamment en quoi elle se distingue d'une simple hybridation des arts. En effet, si le terme d'interdisciplinarité a surtout été évoqué pour faire une place à des pratiques nouvelles et variées, qui n'entraient pas dans le cadre des différents arts, quelque chose de plus sérieux semble se jouer dans les phénomènes d'effrangement: le processus consiste désormais en un emprunt aux structures des autres arts, emprunt dont la visée n'est autre qu'une efficacité prétendument renforcée. Il se distingue donc d'une symbiose ou d'un agglomérat des arts tel qu'il est défini par Wagner dans son projet d'œuvre d'art totale : d'une part, le Gesammtkunstwerk est le nom sous lequel il propose une synthèse des arts ; d'autre part, comme le montre Adorno dans L'Art et les arts, on a affaire, avec l'effrangement moderne, à quelque chose de plus fort, qui bouleverse bien davantage les catégories. Il faudrait, pour lui, être aveugle pour ne pas y voir les signes d'une tendance nouvelle et puissante, tant le phénomène montre de la variété, de l'insistance, et se révèle être bien plus que de l'ostentation ou qu'un retour à cette "synthèse suspecte ", même si, selon lui, on en retrouve des survivances dans les happenings.

\section{Greenberg et Adorno}

Il faut donc saisir et interpréter cet « effrangement des arts ». À cet effet, nous croiserons l'approche critique de Clement 
Greenberg avec l'œuvre de Theodor Adorno, dont les textes sont ici abordés sous le prisme de la problématique de la différence des arts qui, on le verra, n'intervient véritablement chez Greenberg et Adorno que dans un contexte singulier où la notion de genre artistique devient problématique, voire caduque. Notre choix se porte sur ces deux auteurs parce qu'ils sont probablement ceux à avoir le mieux compris que l'art, tel qu'on pouvait le définir depuis plusieurs siècles, était hautement menacé de disparition par le développement soudain du capitalisme industriel au XIXe siècle. À une époque où tout passe par les exigences d'un marché culturel nouveau et ouvert, par les exigences des philistins, le Modernisme doit être une tentative de maintien des normes esthétiques face aux menaces que constituent l'ambiance sociale et matérielle, le caractère des époques, un effort incessant pour endiguer le déclin des normes esthétiques menacées par la relative démocratisation de la culture à l'ère de l'industrialisme, sa logique primordiale et la plus intime. L'un comme l'autre ont saisi le rôle primordial qu'avaient à jouer les avant-gardes dans cette lutte pour la survie de l'art ou, pour reprendre un terme adornien, contre la dés-artisation (Entkunstung) de l'art. En outre, ils sont sûrement les deux théoriciens - avec l'autre grand représentant de la critique formaliste Outre-Atlantique, Michael Fried - dont les analyses de la dislocation des genres artistiques dans les années 1960 furent les plus brillantes et, encore aujourd'hui, les plus fécondes pour interroger les raisons de cette dissolution et tenter d'en comprendre la genèse. Essayons, en premier lieu, de retracer la perspective dans laquelle s'inscrit la mise au travail de la problématique classique de la différence des arts chez Adorno et Greenberg. 
C'est un fait incontestable qu'Adorno et Greenberg connaissaient leurs travaux respectifs. Adorno fait référence à Avant-garde and Kitsch dans la Philosophie de la nouvelle musique; quant à Greenberg, il déclarait à propos d'Adorno : «j'avais une grande estime pour lui. Nous avions les mêmes conceptions à bien des égards. » Par ailleurs, Greenberg a travaillé sur un projet qui réunissait un certain nombre de théoriciens de l'École de Francfort, Studies in prejudice. Rien n'indique, en revanche, qu'ils aient pu avoir une influence réciproque. Rapprocher l'œuvre de Greenberg de celle d'Adorno semble d'ailleurs, de prime abord, assez paradoxal. Il $\mathrm{y}$ a en effet, à première vue, davantage de divergences que de convergences dans leurs conceptions esthétiques. Le premier est un critique d'art kantien et formaliste, à ce titre assez mal préparé pour la théorie de l'art, d'où quelques indéniables erreurs ou insuffisances théoriques de sa part. Le second est le philosophe hégélien qui a peut-être mis en place la dernière esthétique de grande ampleur. Pour autant, et bien qu'ils viennent d'horizons aussi différents, il semble intéressant que tous deux réagissent simultanément et de façon quasiment identique au phénomène qui nous occupe. Dans les années 1960, Adorno entre dans une période particulièrement féconde, où il continue à instruire, sous une forme radicalement fragmentaire, des questions ouvertes de longue main, notamment dans le débat qui commence à l'opposer à l'avantgarde musicale. Dès 1966, il réagit dans L'Art et les arts à l'effondrement des limites entre les arts. Greenberg se sent contraint, alors qu'il s'était jusque-là contenté de son rôle de critique d'art au jour le jour, de glisser sur le terrain de l'esthétique et, dès 1968, de réagir lui aussi à cette nouvelle confusion qui règne dans l'art de son temps, dans un cycle de 
conférences intitulées Seminars. Pourtant, en 1940, Greenberg publiait dans Partisan Review un article qui allait devenir rapidement un des textes majeurs de la critique moderniste. Se réclamant explicitement de Lessing, il y écrivait :

Se laissant guider, consciemment ou inconsciemment, par un exemple de pureté dérivé de l'exemple de la musique, les arts d'avant-garde ont, au cours de ces cinquante dernières années, atteint un degré de pureté et une délimitation radicale de leur champ d'activité sans précédent dans l'histoire de la culture. À présent les arts campent en sécurité, chacun dans ses frontières "légitimes », et le régime du libre-échange a été remplacé par celui de l'autarcie. (1986, p. 32-33 ; je traduis)

Pour Greenberg, les arts d'avant-garde - c'est-à-dire, dans son vocabulaire, ceux qui perpétuent les exigences qualitatives de la tradition - s'étaient engagés dans la voie d'une purification scensée les reconduire à ce qu'ils avaient d'essentiel et d'irréductible, à leur matérialité constitutive. Greenberg tente de mettre en évidence le fait qu'en supplantant la littérature, la musique était à son tour devenue le premier des arts, le parangon de toutes les disciplines; en raison de son caractère «absolu», de son éloignement de l'imitation, dans son absorption quasi totale dans la qualité proprement physique de son médium, grâce également à son pouvoir de suggestion. La musique apparaissait soudain comme l'art abstrait, « la forme pure » par excellence. Dans la suite de ce texte, Greenberg remarque toutefois une certaine confusion déjà présente dans les arts de la première moitié du siècle. Pour autant, ce simple constat ne le préoccupe pas: en effet, il ne concerne alors qu'essentiellement le kitsch.

Mais deux décennies plus tard, lorsqu'il écrit « Modernist Painting », toutes les digues semblent alors rompues, et l'avant- 
garde, qui avait jadis été épargnée, est à son tour menacée par cette tendance. Pour Greenberg, Marcel Duchamp est directement tenu pour responsable de celle-ci, en tant qu'auteur de la démonstration suivante : tout peut désormais être de l'art. Cette affirmation est répétée à de nombreuses reprises :

Depuis les ready-mades de Duchamp, il est devenu plus clair également que tout ce qui peut faire l'objet d'une expérience tout court peut faire l'objet d'une expérience esthétique; et que tout ce qui peut faire l'objet d'une expérience esthétique peut être vu comme art. Bref, art et esthétique ne font pas que se superposer, ils coïncident. (Greenberg, 1976, p. 93 ; je traduis)

Ou encore :

Et pourtant [...] : quelque chose a été démontré qui valait de l'être. De l'art comme celui de Duchamp a montré [...] à quel point même la catégorie de l'expérience esthétique formalisée était ouverte. Cela a toujours été vrai, mais devait être démontré pour être su comme vrai. La discipline esthétique a reçu de nouvelles lumières. (Greenberg, 1971, p. 129; je traduis)

Eu égard aux affirmations précédentes, on peut se poser les questions suivantes : la possibilité que tout puisse faire l'objet d'une expérience esthétique n'est-elle donc pas nouvelle? Cette possibilité réclamait-elle une démonstration? On peut en convenir avec Thierry de Duve: d'une part, les ready-made duchampiens ne constituent en rien des démonstrations; d'autre part, le fait que tout puisse être de l'art n'avait aucunement besoin d'être démontré. Pour Thierry de Duve, on peut le rappeler, le geste duchampien est assimilable à l'envoi d'un message sans auteur, en 1917, qui signifie que tout peut être de l'art et que n'importe qui peut être artiste. Ce message aurait traversé la modernité pour n'être reçu qu'en 1963, avec 
la rétrospective Duchamp à Pasadena ou encore en 1964, date à laquelle des répliques des ready-made ont été produites. N'importe qui peut être artiste depuis le salon des indépendants qui s'est tenu en 1884, marquant l'effondrement de l'autorité du système des Beaux-Arts. Enfin, cet écroulement du système des Beaux-Arts signifie le passage à l'art en général : ce passage, que Thierry de Duve propose de qualifier de "spécifique au générique », s'est joué en peinture, au Salon des refusés de 1863, par le biais de l'invention du non art.

On l'a vu, Greenberg évoque dans ses textes une confusion qui règne dans les arts, mais aussi, et déjà, cette possibilité de faire de l'art sans respecter les contraintes d'un genre. On serait donc tenté de conclure que la possibilité de faire de l'art avec n'importe quoi et d'être artiste sans devenir nécessairement le praticien d'un genre particulier, fasse de l'effrangement adornien une problématique dépassée : Adorno se contenterait en effet d'évoquer une fluctuation des frontières entre les arts les unes dans les autres, une porosité de leurs lignes de démarcation, à une époque où il est d'ores et déjà possible de faire de l'art avec n'importe quoi, autrement dit, en ne respectant plus aucun matériau prédonné, faisant figure de contrainte pour la forme de l'œuvre.

Une telle conclusion est toutefois trop hâtive. Si l'on opère un déplacement par rapport à la manière dont L'Art et les arts, texte parfois abscons, est généralement lu, on peut montrer que ce qu'Adorno nous donne à voir n'est autre que le moment précis d'un long processus, qui s'achève par l'effondrement complet des genres artistiques. Quel est ce processus? Quand commence-t-il et pourquoi? Quel lien entretient-il avec la chute du système des Beaux-Arts? Quelle est cette «force des 
mouvements historiques » qui a fini par submerger les limites entre les différents genres artistiques à l'aube des années 1960 ?

\section{Adorno et L'Art et les arts}

L'Art et les arts a pour vertu incontestable de proposer une analyse de ce qui semble être la dernière étape de la dissolution des genres artistiques, étape qui précède leur éclatement pur et simple et le passage à "l'art en général». Pour Adorno, au début du $\mathrm{XX}^{\mathrm{e}}$ siècle, lorsque les arts s'étaient libérés des contraintes spécifiques à leur genre, ils avaient créé un vide qu'il fallait, d'une manière ou d'une autre, pallier. Les arts avaient alors adopté de nouveaux principes structurels - la libre atonalité schœnbergienne par exemple, à laquelle Adorno reste très attaché, avait ainsi fini par se mouvoir en dodécaphonisme - , adoptant par là-même une nouvelle forme de domination rationnelle.

Mais au début des années 1960, cette vacuité n'est plus comblée par l'adoption de nouvelles règles rigides: il s'agit désormais d'emprunts aux structures des autres arts. On le voit, les « ismes » sont alors remplacés par l'ajout d'adjectifs au mot «art»: art cinétique, art minimal, art conceptuel, etc. Si certains de ces mouvements sont, dans un premier temps, spécifiquement américains - l'art conceptuel et l'art minimal notamment - , le phénomène touche l'Europe comme les ÉtatsUnis. Cette tendance croissante des arts à se nourrir les uns des autres est décrite de manière presque anthropomorphique chez Adorno. On peut risquer l'hypothèse suivante : le phénomène qu'Adorno constate et s'efforce d'interpréter semble correspondre à un chaînon entre les derniers «ismes » 
artistiques du $\mathrm{XX}^{\mathrm{e}}$ siècle et le passage à l'art au singulier. Certaines tendances artistiques de la fin des années 1950 et du début des années 1960 illustrent clairement cette hypothèse. On pourrait faire un rapprochement entre l'effrangement des arts tel que le commente Adorno et ce qui se joue lors des débuts de l'art minimal, en particulier dans la catégorie des Specific Objects créée par l'artiste Donald Judd. Pour les artistes concernés par cette nouvelle catégorie, il était devenu difficile de poursuivre la peinture moderniste dans la voie dans laquelle elle s'était engagée : comment, en effet, aller plus loin que la planéité des toiles noires et monochromes de Stella, marquant, d'une certaine manière, un point final? Comment, pour les musiciens, aller plus loin que le 4'33 de Cage, lui aussi insurpassable dans le sens de la réduction? Comment compenser, en littérature, le retrait du contenu narratif? Pour autant, cette génération d'artistes n'est pas encore prête à travailler dans un genre artistique qui n'ait plus sa spécificité, à la différence d'un Allan Kaprow, pour qui le constat de l'abolition des limites entre les arts s'assimile davantage à un cri de victoire. Progressivement, ces artistes quittent alors la bidimentionnalité pour ajouter à leurs œuvres un élément tridimentionnel, transgressant délibérément la limite de leur genre en tendant vers la sculpture, tout en revendiquant cette transgression. La limite entre les deux arts, qui paraît a priori aller de soi, n'est plus respectée. Il en va de même, nous dit Adorno, des limites entre l'architecture et la sculpture notamment. Un artiste comme Donald Judd reconnaît aux objets spécifiques des qualités à la fois picturales et sculpturales, mais pas leur appartenance à la peinture ou à la sculpture. Ils constituent bien, pour lui, un nouveau genre qui possède sa légitimité, son autonomie, son "aire de compétence propre », 
pour reprendre l'expression greenbergienne. Certes, Adorno n'évoque pas explicitement les artistes concernés par la catégorie des objets spécifiques - Claes Oldenburg, Richard Smith, Dan Flavin, John Chamberlain ou encore Sol Lewitt - et ne participe pas aux débats de ce début de décennie qui visent à statuer sur la légitimité de cette nouvelle catégorie. Cependant, et bien qu'il ne soit pas spécialiste de peinture, ce qu'il perçoit chez Fritz Wotruba et davantage encore chez le peintre allemand Schultze - auteur, dit-il, de «constructions proliférantes »-, n'est pas étranger à la catégorie des objets spécifiques et, plus généralement, aux débuts de l'art minimal. Qu'on lise ces œuvres comme faisant partie de deux genres à la fois ou qu'on considère qu'elles n'appartiennent ni à leur domaine d'extraction (en l'occurrence la peinture, pour ce qui est des objets spécifiques), ni au domaine vers lequel elle tendent-Greenberg et Fried feront le premier choix, discréditant ainsi, en bloc, tout l'art minimal - , force est de constater, d'une part, que les frontières entre les arts se meuvent alors les unes vers les autres et, d'autre part, qu'une visée palliative est indéniable dans cette tendance à l'effrangement. On l'a vu, il s'agit, par exemple, de dépasser la planéité littérale des toiles de Stella en peinture ou encore de compenser le retrait du contenu narratif en littérature par l'adoption de principes issus directement des autres arts. L'Art et les arts semble donc pouvoir être relu de la manière suivante : premièrement, la thèse d'un effrangement des arts met en lumière ce qui semble prendre les traits d'une dernière tentative de pallier les problèmes spécifiques des différents genres tout en continuant à œuvrer dans un art qui ait encore sa légitimité. À ce titre, l'effrangement n'apparaît pas comme la fin d'un processus : il s'assimile davantage à un compromis, à 
une situation transitoire. Deuxièmement, Adorno nous montre que la nouveauté dans ce phénomène récent tient non pas à ce que les arts tendent les uns vers les autres, mais bien au fait que, pour la première fois dans l'histoire de l'art, leurs frontières s'en trouvent atteintes.

Il faut, à ce stade de notre réflexion, revenir aux questions posées plus haut : pourquoi les arts du XXe siècle ont-ils tendu à adopter des principes qui leur étaient extérieurs? Pourquoi, pour la première fois dans les années 1960, leurs frontières s'en trouvent bouleversées, jusqu'à disparaître, ce qui apparaissait inconcevable jusque-là? Les raisons qui ont entraîné la "pseudomorphose» de la musique d'après la peinture, par exemple, sont-elles les mêmes qui aboutissent aux « phénomènes d'effrangement »?

\section{Adorno et le rapport à l'histoire}

Il semble qu'il faille envisager la théorie adornienne sous l'angle de son rapport à l'histoire pour comprendre ce qui se cache dans la tendance à l'effrangement des genres. Dans la Dialectique de la raison, Adorno met en évidence que le processus de civilisation est marqué par une tendance croissante à la rationalisation, qui s'accompagne d'une division du travail toujours plus marquée. On le voit aussi dans les arts : de même que l'homme est scindé socialement par la raison instrumentale, l'art est scindé en arts, les genres particuliers étant ainsi la contrepartie du monde aliéné dans la division du travail. L'explosion des genres signifie, pour les arts, la sortie hors de la dialectique de la raison et de la domination de l'homme par l'homme : elle se présente comme un moment de 
libération sans domination nouvelle. Or, le système des BeauxArts, via l'institution du Salon, était responsable d'une forme de domination en tant qu'il décidait qui pouvait être légitimement artiste et posait les conventions à suivre dans chaque genre particulier.

On peut considérer que l'avant-garde naît progressivement - du XVII e siècle, avec la création du Salon, jusqu'en 1863, date à laquelle est créé le salon des Refusés pour se cristalliser en 1884 avec le Salon des Indépendants et l'effondrement de l'autorité du système des Beaux-Arts. Nous l'avons vu, la dissolution des genres artistiques correspond à une période de libération sans apparition de nouvelles règles rigides: cette libération semble s'être jouée par le biais de l'avant-garde, dès lors que le système des Beaux-Arts est devenu caduque. Le phénomène d'effrangement, repéré dans les années 1960, serait donc l'extension d'une cause antérieure. Comme le montre Adorno, notamment dans Philosophie de la nouvelle musique, ni l'affaiblissement des matériaux ni la tendance à imiter les autres arts ne sont spécifiques aux années 1960. Il y évoque déjà une "pseudomophose de la musique d'après la peinture ", qui s'opère dans la première moitié du siècle. L'existence de tels antécédents témoigne en faveur de l'hypothèse suivante: l'histoire des avant-gardes serait marquée par une reddition progressive, une libération des artistes à l'égard de matériaux prédonnés, qui aboutit finalement à la dissolution complète des genres artistiques. Ainsi peut-on considérer que cette dissolution est déjà contenue, en germe, dans les motivations qui ont engendré la naissance des avant-gardes, comme une tendance inéluctable qui se réalise pleinement au début des années 1960. Mais l'élargissement des matériaux, l'affranchissement vis-à-vis des 
conventions, au sein même des genres artistiques, ne signifie pas encore un passage à l'art "en général » ni même la transgression de leur limites. Pourquoi, soudainement, celles-ci sont-elles devenues poreuses?

Un retour à la pensée greenbergienne pourrait bien éclairer cette nouvelle étape de la dissolution des genres. On le sait, Greenberg tient Marcel Duchamp pour responsable de cette confusion qui règne dans les années 1960. Il fait une erreur théorique en considérant qu'il s'agit d'une démonstration, comme l'a fort justement observé Thierry de Duve; on peut ajouter qu'il en fait une autre en considérant la démarche duchampienne comme essentiellement motivée par l'humour. Mais il est toutefois légitime de penser que Duchamp a bel et bien mis en lumière la chute du système des Beaux-Arts, dont la mort n'a visiblement pas été assimilée par les modernes. Dès lors, la tentation de ne plus respecter la limite entre les genres - dont nous disions qu'elle est déjà présente dès la naissance des avant-gardes en tant qu'elle est une forme de rébellion contre la domination rationnelle et, par là-même, rébellion contre toute forme de division du travail — ne connaît plus aucun frein.

Telles sont probablement les «forces historiques» qu'évoque Adorno dans L'Art et les arts: une tentative croissante de ne plus respecter les territoires établis une fois pour toutes, qui commence par l'affranchissement des œuvres vis-à-vis de leurs matériaux, se poursuit, dès que la mort du système des Beaux-Arts est assimilée, par la dissolution progressive des limites entre les genres et s'achève, peu après, par le passage des arts, au pluriel, à l'art, au singulier. Certes, Adorno n'évoque jamais la responsabilité de Marcel Duchamp, 
pas plus qu'une quelconque influence de celui-ci qui entrerait en jeu dans l'effrangement des genres artistiques. Son nom n'apparaît ni dans la Théorie esthétique ni dans L'Art et les arts. Mais de toute évidence, il existe un rapport d'Adorno à Duchamp, qui se joue via la figure de John Cage, dont certaines des œuvres s'imposent, pour Adorno, comme "phénomènes clef de l'époque » (1995, p. 217). C'est, de toute évidence, une opposition au non-art qui se joue dans le rejet de Cage chez Adorno et le rejet de Duchamp chez Greenberg, dans la mesure où leurs œuvres, ou non-œuvres, sont incompatibles avec leurs propres présupposés. Notons que, comme l'a très bien observé Adorno dans L'Art et les arts, prolongeant son analyse d'une perspective herméneutique, le phénomène de dissolution des lignes de démarcation des genres artistiques a joué un rôle primordial dans l'antagonisme entre les arts contemporains et le public :

Lorsqu'on porte atteinte aux frontières, l'angoisse de défense face au mêlé se réveille facilement [...]. Tout ce qui ne s'en tient pas à la discipline de territoires établis une fois pour toutes passe pour indocile et décadent, alors que ces territoires sont d'origine historique et non naturelle [...]. Face à ses évolutions qu'on estime inconciliables avec le genre artistique au sein duquel elles sont advenues, il y a une forme normale de résistance [...] le brouillage des catégories artistiques bien rangées cause des angoisses civilisées. (Adorno, 2002, p. 6)

Doit-on, aujourd'hui, s'inquiéter que les arts ne travaillent plus dans « le respect du médium » ou encore dans « la contrainte du matériau »? Doit-on penser que la fin d'une différence des arts menace la capacité "sismographique » des œuvres d'art? Ne doit-on pas plutôt passer outre, pour saisir ce qui se joue dans chaque œuvre particulière? Telle était déjà probablement l'entreprise adornienne : le concept philosophique, écrit Adorno 
dans Théorie esthétique, a quelque chose de suranné, tout comme celui d'esthétique ou de morale. L'esthétique, en effet, a constitué son objet à partir de philosophèmes qui sont autant d'invariants et qui répondent à une idéalité première : celle du concept d'art lui-même. Or, précise Adorno, «l'art ne peut être interprété que par la loi de son mouvement, non par des invariants » (1995, p. 17-18). Dès lors, si Adorno conserve le terme d' "esthétique», il lui donne un contenu radicalement nouveau. L'esthétique doit désormais se passer des concepts qui ont perdu toute validité opératoire ; elle doit se construire elle-même à l'œuvre : comme le montre le travail de Benjamin, dont Adorno fait sur ce point l'éloge, toute théorie de l'art ne peut à présent s'élaborer que par le biais d'une immersion dans l'œuvre particulière; il faut pénétrer l'œuvre « comme dans une chapelle », écrit-il, reprenant le mot de Gœthe. Aussi l'enjeu ne semble-t-il pas, aujourd'hui, de former une nouvelle esthétique, dissimulée, et aussi sourde aux œuvres qu'a pu l'être l'esthétique philosophique, mais bien de s'inscrire, de s'engager sur une voie frayée notamment par Adorno, qui est celle d'une pensée de l'œuvre. Pour Christine Eichel, il s'agirait de se donner les moyens d'analyser l'art depuis «l'épuisement des avant-gardes » jusqu'à « l'interconnexion des arts » De se doter d'une esthétique de l'« interdisciplinaire». Toutefois, l'idée d'une interdisciplinarité semble quelque peu dépassée aujourd'hui. Les arts ne se contentent plus, comme l'écrit encore Jean Lauxerois, de jouer à la limite de leurs frontières, rejouant une version à peine dissimulée du drame wagnérien, qui prétendait au rassemblement de tous les arts au service d'une théâtralité alors déjà dénoncée par Mallarmé. Depuis les années 1960, nous le savons, il est possible et légitime d'être artiste sans être nécessairement le praticien d'un art en 
particulier. La limite entre les différents genres a été abolie, et il nous semble qu'Adorno a bien senti, dès la fin des années 1950, que la Modernité faisait déjà partie de l'histoire. Par voie de conséquence, il a affirmé la nécessité de nouveaux modes de considération, de nouveaux paramètres : comment peut-on, en effet, poursuivre une théorie dont l'objet est en radicale mutation?

En renonçant aux définitions solidifiées, Adorno rend possible une essentielle ouverture du regard pour des œuvres d'art qui contredisent à l'évidence son concept de modernité, y compris celles qui sont en train de se faire à l'aube de notre siècle.

\section{L'œuvre d'art, « double » du réel?}

La dissolution, puis l'abolition des genres a entraîné avec elle la limite qui sépare l’art de la vie. Par conséquent, évoquer une autonomie de l'art paraît problématique. C'est avec Adorno, là encore, que nous allons essayer de penser cette question de l'autonomie de l'art et celle du rapport de l'œuvre au réel.

Les arguments adorniens relatifs à la nécessité d'une autonomie de l'œuvre d'art sont souvent assimilés au formalisme de Greenberg. Par ailleurs, la prétendue radicalité des conceptions de Greenberg et d'Adorno, alors jugée similaire, est souvent mise en avant. La forme que revêt l'explication historique de l'autonomie de l'art diffère pourtant chez Adorno et Greenberg : cette différence est précisément ce qui détermine leurs conceptions respectives de l'autonomie de l'œuvre d'art. En effet, pour Greenberg, l'affaiblissement de la religion, de l'«autorité» et de la tradition-comprise ici 
comme la rupture avec les notions desquelles les artistes doivent en grande partie dépendre pour la communication avec leur public - sont les principales raisons de cette autonomie :

On pourrait désormais reconnaître que l'art n'a pas à professer, n'a pas à célébrer ou à glorifier quelqu'un ou quelque chose, n'a pas à promouvoir des causes; qu'il s'est émancipé de la religion, de la politique, et même de la morale. Tout ce qu'il a à faire est d'être bon. (Greenberg, 2003, p. 33 ; je traduis)

Adorno évoque, quant à lui, la forme spécifique que revêt la marchandisation de l'art telle qu'elle apparaît avec le déclin des relations sociales féodales au sein desquelles la production artistique était ancrée. Les deux hypothèses sont indubitablement liées, en tant qu'elles décrivent une facette différente d'un seul et même processus historique; mais ce qui les distingue n'en demeure pas moins crucial. Alors que Greenberg conçoit l'autonomie de l'œuvre d'art uniquement sous l'angle de sa signification, Adorno la conçoit, lui, au moins dans son sens le plus fondamental, sous l'angle des relations sociales constitutives d'un art comme phénomène social. Dans une telle analyse, l'institutionnalisation de l'autonomie rend possible et maintient l'autonomie de l'œuvre. Ainsi, le sens que revêt la notion d' " autonomie » chez Adorno et chez Greenberg n'est pas exactement identique.

La conception de l'esthétique en tant que discipline autonome dans l'œuvre de Baumgarten, puis dans celle de Kant, a été soutenue par le développement de nouvelles formes de relations sociales au XVIIe et au XVIII e siècles, liées à l'émergence de l'individualisme bourgeois. Dans le sillage de la généralisation de ces relations sociales liées à la production de biens, la spécificité théorique de l'esthétique est devenue la base intellectuelle d'une institutionnalisation de l'art en tant 
que marchandise tout à fait singulière : une marchandise dont l'inutilité est à l'origine de la valeur d'échange, dans sa capacité à maintenir une contemplation «esthétique» ou encore "désintéressée». La théorie esthétique acquiert alors une double fonction : à la fois celle de délimiter et de théoriser une forme particulière d'expérience (l'expérience " esthétique ») et celle de réguler et de légitimer la distribution des œuvres d'art au sein du marché, par l'émission de jugements de goût. C'est dans la crise de cette fonction, semble-t-il, - crise aggravée par l'obsolescence toujours plus grande des catégories traditionnelles de l'esthétique pour penser l'art contemporain -, que la signification sociale de la crise survenue aux lendemains de l'abolition des genres artistiques doit être trouvée. Une remise en question fondamentale de l'idée d'autonomie est au cœur de cette crise.

La théorie adornienne a pour vertu de nous permettre de comprendre que cette crise n'est pas uniquement un phénomène historique contingent, mais aussi une étape nécessaire qu'Adorno décrit, d'une manière typiquement hégélienne, comme le développement logique de l'art lui-même. L'œuvre d'art est considérée par Adorno à la fois comme un phénomène social et esthétique : elle est issue du réel tout en s'en détachant. La contradiction entre ces deux caractéristiques de l'art constitue, selon Adorno, « l'essence duelle » de tout art moderne et la dynamique qui sous-tend son développement. En outre, c'est bien la manière de traiter cette contradiction, de manière immanente, par le biais de la forme de l'œuvre d'art particulière, qui détermine son statut d'objet esthétique autonome et sa capacité critique. L'œuvre d'art, chez Adorno, est un produit de la société, son autonomie est toujours engendrée et maintenue par un ensemble spécifique de 
relations sociales, davantage que par un élément qui serait propre à l'objet esthétique en lui-même. Mais en même temps, l'objet artistique s'auto-définit comme étant autonome, se présente lui-même au sein de l'institution comme porteur d'une forme d'expérience spécifique et autonome : l'esthétique. Pour cette raison, les conditions sociales et le réel au sein desquels l'art est produit filtrent dans l'œuvre d'art elle-même bien davantage que comme un simple supplément à sa forme : les antagonismes irrésolus de la réalité réapparaissent en art sous la forme des problèmes immanents de la forme esthétique. C'est bien la façon qu'a l'artiste de traiter ces problèmes, ces « antagonismes irrésolus » du réel, de manière immanente, qui détermine si chaque œuvre d'art singulière peut être jugée véritablement autonome. C'est donc sous la forme d'une contradiction entre le statut de marchandise de l'œuvre d'art et son statut d'objet esthétique autonome que l'essence contradictoire de l'art, dans les sociétés capitalistes, se manifeste et se comprend. La forme que revêt cette contradiction peut être comprise comme celle de la contradiction entre deux aspects de la marchandise elle-même : la marchandise comme fétiche et la marchandise pourvue d'une valeur d'échange. Ce second aspect a créé les conditions pour la subversion du premier, en réduisant la valeur esthétique de l'objet aux valeurs du marché, via la pression exercée sur la production des œuvres d'art par les facteurs idéologiques et commerciaux au travail dans les différents réseaux de leur distribution et de leur consommation. Ce qui explique à la fois la dynamique de la dialectique du modernisme et l'inintelligibilité ou l'hermétisme croissant des œuvres d'art « authentiques » du modernisme tardif. Pour Adorno, l'histoire de la modernité est, à son origine, celle des stratégies par le 
biais desquelles l'œuvre d'art a résisté à sa propre forme sociale dans le but de pouvoir continuer à révéler le véritable sens de l'ordre social dont elle fait partie, comme une forme de nonliberté. Il apparaît clairement que l'autonomie esthétique de l'œuvre d'art est à la fois ancrée dans son autonomie institutionnelle et finalement dépendante de celle-ci, mais la forme spécifique de l'institutionnalisation de cette autonomie (la marchandisation) n'en demeure pas moins une menace pour l'œuvre. Il semble également indéniable que le statut autonome de chaque œuvre particulière doit toujours être jugé en fonction de sa capacité immanente à résister aux valeurs du marché, au sein duquel il doit cependant acquérir sa réalité sociale. Toute œuvre d'art singulière peut seulement être jugée véritablement, authentiquement autonome lorsque la logique de sa production reste indépendante des valeurs extraesthétiques. Cela ne signifie pas pour autant que l'œuvre d'art ne peut être compatible, dans une certaine mesure, avec une partie de ces valeurs : elle doit en effet l'être pour acquérir une existence indépendante en tant qu'œuvre d'art au sein de l'institution. C'est au dilemme contenu dans ce paradoxe que doit faire face tout artiste contemporain lorsqu'il est confronté aux problèmes relatifs à la forme. Et c'est dans la lutte de l'artiste contre ce problème que les antinomies sociales se transforment en dialectique des formes et que, dans sa manière d'articuler ces antinomies dans le langage de l'art, l'artiste joue son rôle social distinctif. Le statut de l'art est donc antinomique et rien ne semble permettre de résoudre cette antinomie : la tentative de la résoudre soit par l'art engagé, soit par une réintroduction de l'art dans le réel ne signifie qu'une liquidation de la substantialité de l'art, du moment de résistance que contient cette antinomie. 
On peut dès lors affirmer que l'autonomie de l'art moderne, telle qu'Adorno nous aide à la concevoir, possède trois sens distincts et implicites. Premièrement, l'art est autonome de manière "théorique », en tant que lieu privilégié d'une forme particulière d'expérience. Deuxièmement, il est socialement ou encore institutionnellement autonome, dans la mesure où les normes et les pratiques des institutions du monde l'art permettent la réalisation de cette autonomie théorique par le biais d'une forme distincte d'expérience sociale: l'expérience esthétique. Enfin, il est autonome de manière immanente, dans la mesure où la logique de production de l'œuvre se conforme à celle de la production d'un objet à même d'engendrer, chez le regardeur, une expérience esthétique «autonome». Or, dans les écrits de Greeberg, ce deuxième sens apparaît totalement absent, tandis que le troisième s'avère considérablement réduit au premier dans l'idée de «valeurs» spécifiquement esthétiques, dans celle d'une expression «pure» des «éléments irréductibles de l'expérience », ou encore dans la réduction du sens de l'œuvre aux propriétés formelles de son médium. Greenberg précise cependant, à plusieurs reprises, qu'il n'a jamais utilisé le terme de pureté sans guillemets. Chez lui, semble-t-il, l'idée d'une autonomie devient alors inextricablement liée à elle d'une autoréférentialité. Une telle notion est absente chez Adorno, qui montre que la fonction première de l'idée est de dénier un quelconque rôle constitutif au social dans l'esthétique. Greenberg est certes conscient de l'existence de ce qu'il nomme « un cordon ombilical d'or » (umbilical cord of gold) qui relierait l'artiste moderne à la société : pour lui, aucune culture ne peut se développer sans une base sociale, sans une source de revenu stable. Et dans le cas de l'avant-garde, celui-ci a été fourni par 
une élite appartenant à la classe dirigeante dont elle s'est considérée comme séparée, mais dont elle est restée attachée par ce "cordon ombilical d'or». Mais Greenberg s'en tient néanmoins à définir ce lien en termes de dépendance, négligeant par là même le fait que c'est par le biais de la forme spécifique de cette dépendance (sa relation au marché) que l'art moderne devient autonome. La divergence d'Adorno et de Greenberg sur ce point précis semble à l'origine de leur conception différente de l'œuvre d'art moderne. Alors que, chez Greenberg la notion d'autonomie semble essentiellement tributaire du degré de purification de l'œuvre d'art par ellemême - purification qui s'opère par le rejet de tout contenu extra-esthétique, c'est-à-dire extérieur aux propriétés formelles de son médium : pour la peinture, il s'agit d'exclure la narration, le récit, la «littérature» qui «infeste» la peinture avec ses « contenus idéologiques»-,chez Adorno, une telle notion de pureté est inenvisageable et indésirable: l'enjeu n'est pas de déterminer si une œuvre s'est ou non libérée des conditions sociales de sa production, mais bien plutôt de savoir si elle est parvenue à donner à ces conditions sociales contradictoires une expression esthétique " authentique » ou encore " autonome ». Le sens qu'Adorno et Greenberg donnent à l'abstraction, notion centrale de leur théorie, semble pouvoir illustrer cette différence de conception. Pour Greenberg, qui évoque les moyens par lesquels le médium est purifié, débarrassé de tout contenu extra-esthétique, l'abstraction est un élément formel essentiel de l'autonomie. Chez Adorno, elle est un reflet de la tendance croissante à l'abstraction caractéristique de toutes les relations sociales au sein des sociétés capitalistes ainsi qu'une "stratégie» esthétique historique visant à exprimer la réification qui en résulte. Par conséquent, pour Adorno, il n'y a 
pas de lien direct, a priori, entre l'abstraction et l'autonomie de l'œuvre. Ainsi, si l'on le suit, les œuvres du réalisme socialiste, comme toutes les œuvres d'art dites «engagées», ont un caractère réactionnaire (d'où le tort de Brecht de prendre directement le thème de la lutte des classes pour la création de ses pièces de théâtre), adoptant un discours apologétique d'une pesanteur indéniable, didactique et sermonnaire : ce type d'art tombe donc inéluctablement dans un autoritarisme identique à celui qu'il essaie de dénoncer; il se trouve toujours au-dessous de la réalité des conflits, les figeant dans leur mouvement et les schématisant à l'extrême :

[Le programme brechtien] de distanciation était de provoquer la réflexion $\mathrm{du}$ spectateur. Le postulat brechtien d'un comportement réflexif converge remarquablement avec celui d'une attitude de prise de conscience, objectif qu'attendent les œuvres d'art autonomes et importantes de la part des spectateurs, des auditeurs et des lecteurs, comme étant l'attitude adéquate. Son gestus dialectique est cependant intolérant envers la pluralité d'interprétation au contact de laquelle la pensée s'enflamme : il est autoritaire. (Adorno, 1995, p. 321)

Le didactisme, l'intolérance, le pragmatisme placent toute création «engagée » aux antipodes de l'art véritable, auquel il suffit d'exister pour être révolutionnaire; sa seule présence, désintéressée, est déjà une critique des systèmes totalitaires :

Toutes les grandes œuvres d'art, même les plus affirmatives, sont a priori polémiques. L'idée d'une œuvre d'art conservatrice contient quelque chose d'absurde. En se séparant de manière emphatique du monde empirique, de leur autre, les œuvres d'art témoignent que ce monde lui-même doit devenir autre chose, schémas non-conscients de la transformation. (Adorno, 1995, p. 235-236) 
De ce texte il ressort que l'expression « art révolutionnaire » est contradictoire ou qu'elle est tout simplement un pléonasme, l'art étant le seul domaine échappant aux structures totalitaires, aux contraintes extérieures. Pour Adorno, tout engagement est condamnable puisqu'au lieu de servir les intérêts d'une révolution, l'œuvre risque de s'exposer à l'emprise du totalitarisme, de se présenter comme « une bombe par avance désamorcée ». La critique n'est jamais la meilleure lorsqu'elle dénonce un contenu social manifeste ; elle acquiert au contraire toute sa force et sa violence quand ce contenu est camouflé, intégré à la forme. Dans ses Notes sur la littérature, Adorno se fait une fois encore défenseur de Beckett, l'un des seuls dont les œuvres « acosmiques » puissent être qualifiées d'engagées. Il y écrit :

Les philosophes apologistes [des œuvres de Beckett] ont beau y voir un projet anthropologique, il s'agit là de faits historiques des plus concrets: la démission du sujet. L'ecce homo de Beckett, c'est ce que les hommes sont devenus. Ils nous regardent sans mot dire, à travers ses phrases, de leurs yeux secs à force d'avoir pleuré. La fascination qu'ils exercent sur nous et qui s'exerce sur eux se rompt en se reflétant sur eux. Pour cette minuscule promesse de bonheur qui ne se perd pas en consolations, il a certes fallu payer un prix élevé : aller jusqu'au bout de l'articulation jusqu'à perdre tout contact avec le monde. Il faut dénoncer tout engagement en faveur du monde pour satisfaire à l'idée de l'œuvre engagée. (1999, p. 301)

Si l'art doit donc exclure par principe l'engagement, il n'est pas pour autant une fuite hors du monde, une consolation pour les «esprits graves» que nous serions (Nietzsche), une «douce narcose » (Freud). Il est une "critique de la société par sa simple présence [et] son caractère social est la négation déterminée de la société déterminée » (Adorno, 1995, p. 335) : 
sa fonction sociale étant paradoxalement une absence de fonction, car c'est en se différenciant du réel que les œuvres expriment, de manière négative, la promesse d'un monde autre.

\section{Bibliographie}

ADorno, Theodor W. (1985 [1962]), Philosophie de la nouvelle musique, traduction Alex Linderberg et Hans Hindelbrand, Paris, Gallimard.

-. (2002 [1966]), L'art et les arts, traduction Jean Lauxerois, Paris, Desclée de Brouwer, coll. « Art et esthétique ».

—. (1995 [1970]), Théorie esthétique, traduction Marc Jimenez, Paris, Klincksieck, coll. « Esthétique ».

—. (1999 [1958]), Notes sur la littérature, traduction Sybille Muller, Paris, Flammarion, coll. «Champs Flammarion sciences ».

—. et Max Horkeimer. (1974 [1947]), La Dialectique de la raison, traduction Eliane Kaufholz Paris, Gallimard.

DE DuVE, Thierry. (1989), Au nom de l'art, pour une archéologie de la modernité, Paris, Minuit, coll. «Critiques».

GreEnBeRG, Clement. (1986 [1940]), «Towards a Newer Laocoon », dans The Collected Essays and Criticism, vol.1, Chicago, University of Chicago Press. 
-. (2003[1976]), "Seminar Six», dans Late Wrtings, St Paul, University of Minnesota Press, p. 74-86.

-. (2003 [1971]), "Counter Avant-garde », dans Late Wrtings, St Paul, University of Minnesota Press, p. 5-19.

-. (1965 [1961]), "Modernist Painting », Art \& Literature, vol. 4, p. 193-201.

-. (2003 [1980]), "Modern and Postmodern», dans Late Writings, St Paul, University of Minnesota Press, p. 25-34.

KosuTH, Joseph. (1991[1969]), "Art After Philosophy », dans Collected Writings, 1966-1990, Cambridge, MIT Press.

MARCuSE, Herbert. (1973 [1972]), Contre révolution et révolte, traduction Didier Coste, Paris, Seuil, coll. « Combats ».

OBALK, Hector. (2001[1990]), Andy Warhol n'est pas un grand artiste, Paris, Flammarion, coll. « Champs ».

RosenBerg, Harold. (1991[1972]) La Dé-définition de l'art, traduction Christian Bounay, Paris, Jacqueline Chambon.

—. (1964), The Anxious Object, New York, Horizon, 1964.

\section{Résumé}

L'effondrement des limites entre les genres artistiques entraîne celui des limites qui séparent l'art de la vie. Poser l'autonomie de l'art devient alors problématique : un tel énoncé suppose en effet l'indépendance de l'art vis-à-vis du réel, de tout ce qui lui est extrinsèque et n'obéit pas à ses règles propres. D'où l'intérêt 
d'une analyse de la conception adornienne de l'autonomie de l'art, conception particulièrement riche et féconde pour penser le rapport de l'art au réel, dans la mesure où le souci de sauvegarder l'autonomie de l'art - la distance qui la sépare du réel et de la vie - tout en conservant son aspect social y est constant. Sa fonction sociale étant paradoxalement une absence de fonction, c'est en se différenciant du réel que les œuvres expriment, de manière négative, la promesse d'un monde autre.

\begin{abstract}
The collapse of boundaries between artistic genres leads to the collapse of the boundaries that separate art from life. Stating the autonomy of art thus becomes problematic: such a statement presupposes the independence of art vis-à-vis the "real", vis-à-vis all that is extraneous and does not obey its own rules. Hence the interest of an analysis of Adorno's conception of the autonomy of art, particularly rich and fruitful to think the relation of art to reality in its desire to safeguard the autonomy of art - the distance that separates it from the "real" and from life - while retaining its social aspect. Its social function being paradoxically a lack of function, it is in differentiating themselves from the "real" that the works express, in a negative way, the promise of another world.
\end{abstract}

